\title{
Technology Innovation Enabling Falls Risk Assessment in a Community Setting
}

\author{
Cliodhna Ni Scanaill • Chiara Garattini • \\ Barry R. Greene • Michael J. McGrath
}

Published online: 7 December 2010

C The Author(s) 2010. This article is published with open access at Springerlink.com

\begin{abstract}
Approximately one in three people over the age of 65 will fall each year, resulting in significant financial, physical, and emotional cost on the individual, their family, and society. Currently, falls are managed using on-body sensors and alarm pendants to notify others when a falls event occurs. However these technologies do not prevent a fall from occurring. There is now a growing focus on falls risk assessment and preventative interventions. Falls risk is currently assessed in a clinical setting by expert physiotherapists, geriatricians, or occupational therapists following the occurrence of an injurious fall. As the population ages, this reactive model of care will become increasingly unsatisfactory, and a proactive community-based prevention strategy will be required. Recent advances in technology can support this new model of care by enabling community-based practitioners to perform tests that previously required expensive technology or expert interpretation. Gait and balance impairment is one of the most common risk factors for falls. This paper reviews the current technical and nontechnical gait and balance assessments, discusses how low-cost technology can be applied to objectively administer and interpret these tests in the community, and reports on recent research where body-worn sensors have been utilized. It also discusses the barriers to adoption in the community and proposes ethnographic research as a method to investigate solutions to these barriers.
\end{abstract}

Keywords Gait · Balance · Community assessment · Falls risk

As the global population ages and care ratios (the ratio of those typically in the labor force to those typically not in the labor force, e.g. children and older adults) decline (United Nations Department of Economic and Social Affairs 2009), it is imperative in

C. Ni Scanaill $(\bowtie) \cdot$ B. R. Greene $\cdot$ M. J. McGrath

Health Research \& Innovation, Intel Labs, Leixlip, Co. Kildare, Ireland

e-mail: Cliodhna.Ni.Scanaill@intel.com

C. Ni Scanaill • C. Garattini • B. R. Greene $\cdot$ M. J. McGrath

Technology Research for Independent Living (TRIL) Centre, UCD CASL, Belfield Park Unit 8, Clonskeagh, Dublin 4, Ireland 
this context that health care systems adapt to reduce the incidence and severity of preventable conditions. Falling is one such condition. Falls occur in one of three older adults, and the incidence and frequency of falling increases with age (Kenny et al. 2009). Falls have both societal and economic costs. A recent study calculates that that the mean cost of an injurious fall ranged from US \$3,476 per faller to US \$10,749 (Davis et al. 2010a, b), and falls are the leading reason for admission to nursing homes (Masud and Morris 2001). If falls rates are not reduced in the immediate future, the number of injuries caused by falls is projected to double by 2030 (Kannus et al. 2007). Falls, even non-injurious falls, can affect the faller and their family's confidence. The faller may develop fear of falling (Tinetti et al. 1990), a condition which typically results in the faller self-limiting their physical and social activity in an attempt to avoid further falls. However, in reducing activity, the faller can reduce their aerobic, muscular, and cognitive fitness, thus increasing their risk of further falls.

Despite many years of research, the mechanisms of falls are still poorly understood (Kenny et al. 2009). This is due to the fact that most fallers only interface with the health care system following an injurious fall; thus, the root cause of their fall is investigated retrospectively by taking a falls history (difficult when the fall is accompanied by loss of consciousness) and by recording some physiological measures (which may not identify intermittent causes of falls, such as arrhythmias). However, there are many well-conducted clinical trials examining intrinsic and extrinsic commonalities between fallers and non-fallers. A recent Cochrane review (Gillespie et al. 2009) examined the findings from these studies and identified that fallers typically exhibit one or more of the following risk factors:

- previous falls

- impaired gait andlor balance

- fear of falling

- age and gender

Some risk factors are modifiable and some, such as age and gender, are not. As the risk of falling increases according to the number of risk factors present, it is important to screen for (multi-factorial assessment) and treat (multi-factorial intervention) as many risk factors as possible. Gait and balance impairment is one of the most common risk factors for falls, and intervention through gait and balance retraining has been shown to be an effective method to reduce the incidence of future falls (Davis et al. 2010a, b). Gait and balance impairment was identified (along with recurrent falls, injurious falls, and fear of falling) by the recent AGS $\backslash$ BGS Falls Prevention Guidelines (2010 (online)) as a gateway screening measure to identify potential fallers in the community. This guideline recommends that all adults who are over 65 should be screened annually for falls risk, and if they meet any of the falls risk-screening criteria, they should be referred for full multi-factorial assessment.

\section{Identifying Fallers and "At-Risk" Individuals}

There are many falls algorithms in clinical literature (American Geriatric Society, et al. 2010 (online); Health Service Executive 2010; National Institute for Clinical 
Excellence (NICE) 2004), all of which have the same fundamental structure. The first step of each algorithm describes population-based screening of falls and falls risk. This step usually occurs where the faller first interfaces with the health care system, such as a community clinic, residential care, or acute hospital. If the older adult is deemed to be at risk of falling, they are referred to the second step: multifactorial falls risk assessment. Experienced falls clinicians assess multi-factorial risk factors in a dedicated falls clinic. The final step of any algorithm is the prescription of an appropriate multi-factorial intervention. The experienced clinicians in the falls clinic may prescribe the intervention (e.g. modification of drugs), or they may refer the faller to a specialist for additional assessment (e.g. syncope) and intervention. Figure 1 illustrates the falls algorithm developed by the Health Service Executive (HSE) in Ireland.

\section{Community-Based Screening}

It is widely expected that most falls could be prevented if falls risk factors were identified and treated in a timely manner. It is simple to investigate falls history and falls risk if an individual seeks medical help for a fall-related injury. However, it is a

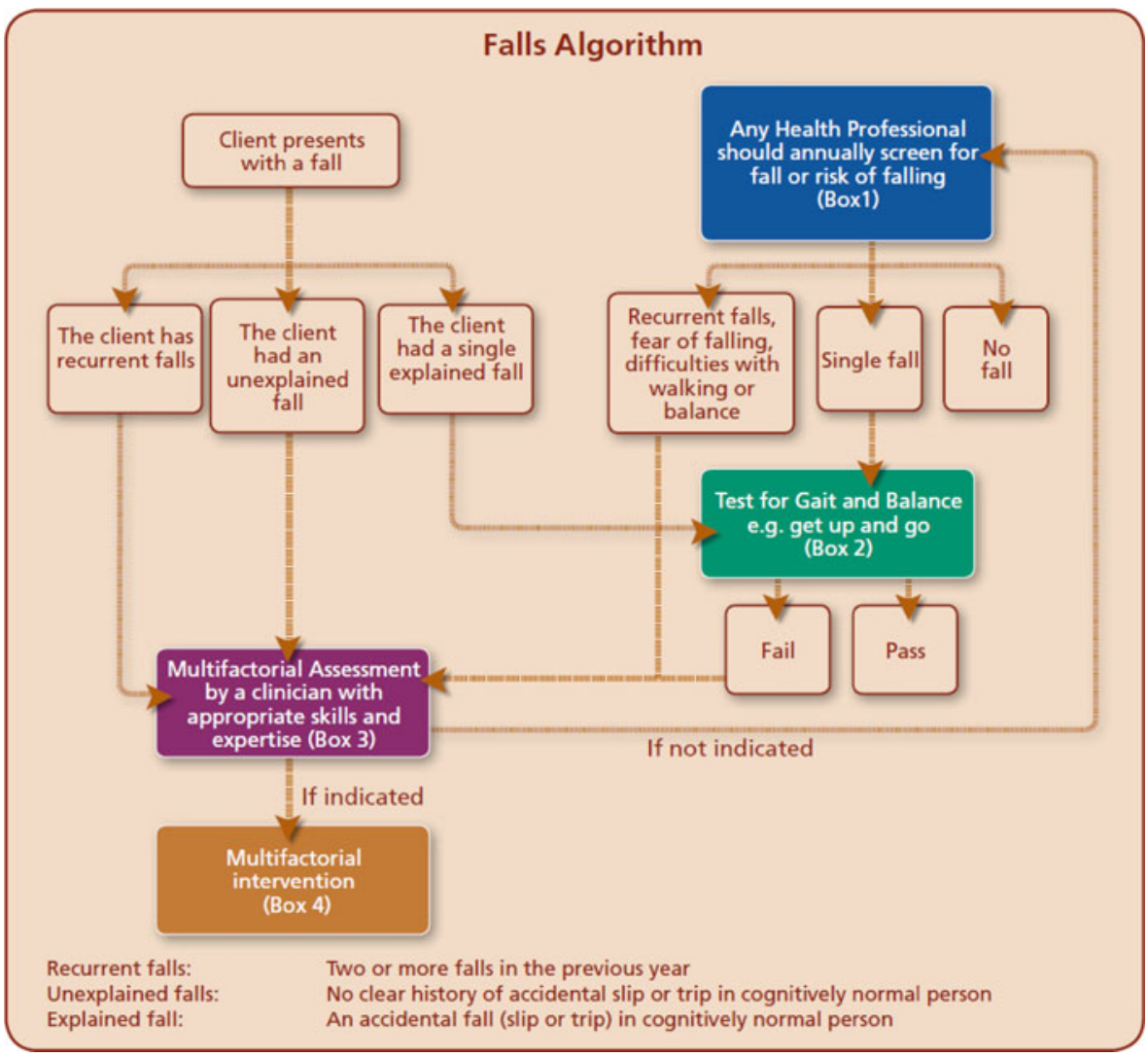

Fig. 1 Falls algorithm developed by the Health Service Executive, Ireland (2010) 
much more complicated task to investigate falls history or falls risk in those who present to a doctor for a non-fall related condition. The clinician must ensure that they not only get a full falls history, if one exists, but they must also evaluate gait and balance, and medically assess the patient for the condition for which they sought medical attention, all during a limited period of time. They must apply their subjective clinical expertise to decide if the older adult's falls history suggests a risk of future falls and warrants specialist multi-factorial evaluation.

Physiological measures, such as gait and balance, should be simple to measure objectively and consistently. Unfortunately, gait and balance are often observed or measured using subjective scales. Time is critical in a community setting, therefore quickly administered functional performance scales such as Timed Up and Go (TUG) (Podsiadlo and Richardson 1991), Functional Reach Test (Duncan et al. 1990), and Turn $180^{\circ}$ (Fitzpatrick et al. 2005) are commonly used to screen for gait and balance impairment. These scales have quantifiable scores (time, distance, and step count respectively) and are repeatable, thus improvement or decline can be monitored longitudinally. However, these tools simply identify falls risk without diagnosing why a person is at risk, therefore making it difficult to determine specific interventions. The thresholds used to assess those at risk of falling are quite broad and they simply identify the high and low end of the falls risk spectrum. Thus, there is a large undifferentiated mid-range score in which most individuals are distributed, and the risk of falling must be subjectively judged by the clinician. Ordinal scales, such as the Berg Balance Scale (Berg et al. 1989), BESTest (Horak et al. 2009), and POMA (Tinetti 1986), can offer more detailed assessment and be of diagnostic value to a person's specific falls risk factors, but these scales take a minimum of $10 \mathrm{~min}$ to administer and are thus unsuitable for general population screening.

\section{Falls Risk Assessment in Falls Clinic}

Falls clinics assess individuals who have already fallen or that are at high end of the risk spectrum. It is at this late juncture that detailed tests for falls risk factors are performed on patients who have already fallen or are at high risk of falling. Falls clinics are staffed by specialist geriatric doctors, nurses, and physiotherapists who perform comprehensive evaluations of falls risk. They have the time to thoroughly investigate falls history and to administer lengthy scales and assessments, which would not be feasible in a community or acute setting. As a specialist clinic, they have the throughput and budgets to justify purchasing expensive diagnostic equipment. Although multiple risk factors are assessed in a falls clinic, only those that examine gait and balance will be considered here.

\section{Balance Assessment}

Impaired balance is common in older adults, and is a well-established falls risk factor (Gillespie et al. 2009). Balance is impaired in three ways as we age: first, the sensory inputs which inform the balance systems become less sensitive as we age; second, the ability of the brain to quickly integrate information from the balance system and react are impaired; and third, the ability of the musculoskeletal systems to maintain 
balance and react quickly to balance disturbances become impaired due to muscle weakness and injury. These age-related deteriorations can be further exacerbated by illness and disease. Balance is typically assessed in a falls clinic through a series of tasks that challenge the person's static and dynamic balance. If their balance is found to be impaired, the cause of their impairment is investigated further in the falls clinic, or they are referred for further specialist evaluation.

Ordinal scales (Tyson and Connell 2009), such as the Berg Balance Scale, the Performance-Orientated balance and Mobility Assessment (POMA), or BESTest are the most commonly used methods to assess balance. These assessments require the older person to perform a series of tasks that require steady-state or anticipatorypostural control. Their ability to complete these tasks is observed and subjectively rated by a clinician. Functional performance scales such as Timed Up and Go (TUG), Functional Reach Test, and Turn $180^{\circ}$, are also used in Falls Clinics.

Technology-based solutions are becoming increasingly common in falls clinics. Pressure sensitive floor mats, such as the GaitRite M2 (2010) (www.gaitrite.com), Tekscan Sway Analysis Module and Sensors (2010) (www.tekscan.com), and Novel Pedar (2010) (novel.de), measure pressure distribution under the feet and deviations in centre of pressure, while the person performs a series of tasks. Some companies, such as Biodex (www.biodex.com), have developed specialized fall-risk screening tools (e.g. the Biodex Falls Screening and Conditioning Program 2010) that are used in association with their existing balance-measuring sensors, but the most commercial simply calculate a number of pressure-based parameters, thus leaving the study design and interpretation of the parameters to the clinician or researcher.

Force-plates (AMTI Force Platforms 2010) are costly but highly accurate instruments that measure three-dimensional ground reaction forces generated by a body standing on or moving across them. They must be installed in a specialist environment and require experts to administer the motion analysis tests and interpret the results. They are typically too costly for use in standard falls clinics, but falls clinics may refer patients for evaluation on a force plate if they observe balance impairment that warrants detailed evaluation. Patients may also be referred for posturography if the source of the balance impairment is unclear. Posturography (NeuroCom EquiTest ${ }^{\circledR}$ 2010) (www.resourcesonbalance.com) is used to objectively identify abnormalities in the three sensory systems (visual, vestibular, somatosensory) and balance processing system by challenging each of these systems individually and measuring the impact of these challenges on anterior-posterior sway and response time. Complex and bulky equipment is required for posturography - a moveable force place, variable stimuli, and a tethering harness and frame to prevent a person from falling; therefore, posturography is considered too expensive, cumbersome, and time consuming for all but the most detailed balance evaluation or research environments.

Low-cost, light-weight, Body Sensor Networks (BSN) are increasingly being applied for gait and balance assessment. Unlike the floor sensors discussed earlier, BSNs are portable and can therefore be deployed in a variety of clinical and nonclinical settings, including a doctor's office or in the home. BSNs can consist of one or a combination of the following wearable sensors: accelerometers, gyroscopes, magnetometers, goniometers, or insoles - and a data-logging, data-transmission, or data-processing aggregator. A brief description of each sensor can be found in 
Table 1 Sensors used in body sensor networks

\begin{tabular}{ll}
\hline Sensor & Description \\
\hline Accelerometers & $\begin{array}{c}\text { measure acceleration, velocity, and displacement of the body segments to which } \\
\text { they are attached } \\
\text { sense angular velocity, are commonly employed on their own or with } \\
\text { accelerometers to measure rotation of the body during motion }\end{array}$ \\
$\begin{array}{l}\text { Gyroscopes } \\
\text { mector } \\
\text { magnetometers } \\
\text { Goniometers }\end{array}$ & $\begin{array}{l}\text { measuring the spatial orientation of the limb to which they are attached } \\
\text { aressure-sensitive } \\
\text { insoles }\end{array}$ \\
$\begin{array}{c}\text { captures foot contact time, pressure distribution under foot, and centre of pressure of } \\
\text { each foot }\end{array}$
\end{tabular}

Table 1. BSN sensors, with the exception of insoles, can be attached to various body segments to measure the balance control and compensatory tactics (such as outstretched hands) of the entire body.

Balance assessment using BSNs is mainly performed in research environments, as most BSNs simply record raw sensor data that must then be interpreted. The McRoberts Dynaport Sway Test (2010) (www.mcroberts.nl) measures RMS, sway angle, and eccentricity from a single body-worn kinematic sensor. Tekscan F-Scan (2010) insoles (www.tekscan.com) can also be combined with the Tekscan Sway Analysis Module (SAM), to measure sway parameters.

\section{Gait Assessment}

Gait assessment has been shown to be useful in assessing the health of the neuromuscular system in a variety of populations, including: Parkinson's disease ("rushed gait" and freezing), stroke (asymmetric gait and drop foot), musculoskeletal injury such as arthritis (asymmetric gait), and fear of falling ("cautious" gait). Gait impairments, like balance impairments, occur due to impaired sensory inputs, impaired processing ability, and musculoskeletal impairments. Gait is a challenging balance task that requires transfer of balance from one foot to another in order to progress forwards. As visual acuity, depth perception, and peripheral sensation become impaired, the ability to predict potential hazards decreases and the potential for stumbling and falling increases. As we age, gait becomes less automatic and brain function previously used to perform secondary tasks, such as carrying laundry, must be shared for gait and the secondary function, resulting in slower more unsteady gait.

Gait is assessed in numerous ways, ranging from observation or simply recording the time taken to walk a specific distance in a community setting, to optical-motion analysis in a specialized gait lab. To accurately measure gait, it is necessary to quantify gait velocity, the location of feet relative to each other (spatial parameters), the timing of footfalls (temporal parameters), and the variability of these parameters. The ability of an individual to perform secondary tasks while walking is also assessed, as single-task walking in a clinical environment is often not truly representative of the physically and cognitively challenging environment leading to a fall in the home (Zijlstra and Aminian 2007). Community-based screening is 
preferable to clinical assessment of gait, as these measurements can be influenced by the "white coat" effect (Table 2).

The "gold standard" method for measurement parameters of human gait is considered to be the force-plate (Davis et al. 2010b) and/or optical motion capture systems (Codamotion 2010). These systems are costly and require a permanent laboratory setting with specially trained personnel to run them. Gait walkways and gait mats require minimal training to use and are quick to set up and record data, and are thus commonly found in falls clinics. Although these devices can be moved, they are not particularly portable. BSNs are inherently portable and have the ability to record objective data in non-tethered conditions. Thus, the protocol or location of a clinical-gait assessment using BSNs can be easily altered without moving or purchasing additional equipment.

\section{Research Trends}

Researchers have found that gyroscope-based temporal gait algorithms have compared favorably to gold-standard methods of gait analysis (Aminian et al. 2004; Salarian et al. 2004) and have recently developed algorithms to measure spatial gait parameters using BSNs (Aminian et al. 2002). BSNs have been shown (Giansanti 2006; Narayanan et al. 2010; O'Sullivan et al. 2009) to produce comparable results to existing clinical balance scales (Tinetti Scale, Physiological Profile Assessment, and Berg Balance Scale and TUG respectively). One such test, the Timed Up and Go test (TUG), has recently received attention from BSN researchers. The TUG is a quick, popular, and highly predictive gait and balance assessment tool that has been shown to have some predictive power for falls risk assessment. The TUG requires a person to rise from a chair, walk $3 \mathrm{~m}$, turn, walk

Table 2 Sensors used for gait and balance assessment

\begin{tabular}{|c|c|c|}
\hline Sensor & Description & Example \\
\hline Force Plate & $\begin{array}{l}\text { measures the ground reaction forces generated by a } \\
\text { body standing on or moving across them }\end{array}$ & $\begin{array}{l}\text { AMTI Force Platforms } \\
2010 \text { (amti.biz) }\end{array}$ \\
\hline $\begin{array}{l}\text { Optical Motion } \\
\text { Capture } \\
\text { Systems }\end{array}$ & $\begin{array}{l}\text { Motion capture units track markers applied to } \\
\text { anatomical landmarks on the body, to compute the } \\
\text { underlying motion of the bones during gait. }\end{array}$ & $\begin{array}{l}\text { CODAMotion }(2010) \text { (www. } \\
\text { codamotion.com) }\end{array}$ \\
\hline Gait Walkway & $\begin{array}{l}\text { long (3-5 meter) pressure sensitive walkway that } \\
\text { measures static and dynamic pressure and force } \\
\text { measurements over several steps using a low profile } \\
\text { floor walkway }\end{array}$ & $\begin{array}{l}\text { GaitRite Walkway (2010) } \\
\text { (www.gaitrite.com) }\end{array}$ \\
\hline Gait Mat & $\begin{array}{l}\text { short }(<1 \text { meter) low-profile floor mat that captures } \\
\text { dynamic pressure as person walks over it }\end{array}$ & $\begin{array}{l}\text { Tekscan MatScan (2010) } \\
\text { (www.tekscan.com) }\end{array}$ \\
\hline Insoles & $\begin{array}{l}\text { in-shoe pressure sensors that capture dynamic and } \\
\text { force information to measure the interaction between } \\
\text { foot and footwear }\end{array}$ & $\begin{array}{l}\text { Tekscan F-Scan (2010) } \\
\text { (www.tekscan.com) }\end{array}$ \\
\hline BSNs & $\begin{array}{l}\text { Wearable sensor(s) that consist of one of a } \\
\text { combination of accelerometers, gyroscopes, } \\
\text { magnetometers, and foot switches to measure } \\
\text { temporal and spatial parameters of gait }\end{array}$ & $\begin{array}{l}\text { McRoberts Dynaport Gait Test } \\
\text { (2010) (www.mcroberts.nl) }\end{array}$ \\
\hline
\end{tabular}


$3 \mathrm{~m}$ and sit down as quickly as is safely possible. If the person takes longer than $14 \mathrm{~s}$ to complete the test, they are judged to be at high risk of falling within 1 year (Shumway-Cook et al. 2000). Researchers have applied BSNs to quantify the various components of the TUG and investigate if some components have a stronger association with falls risk than others or than the TUG itself. Najafi et al. (2002) used tri-axial accelerometer recordings made during the sit-stand and stand-sit transitions to provide a quantitative assessment of falls risk in 11 elderly patients. Skrba et al. (2009) used automatically extracted video-based parameters to quantify turning and walking in 63 elderly adults while performing the TUG test. Similarly, in a recent study of 349 patients, Greene et al. (2010) found the quantitative assessment of the TUG retrospectively estimated falls risk in elderly patients more effectively than either the manually recorded time to complete the TUG test or the Berg Balance Scale.

Falls researchers have shown interest in the Nintendo Wii Balance Board, following its adoption by older adults for gaming and fitness (Clark et al. 2010), compared the Wii Balance Board (WBB) to a laboratory-grade force-plate and found that the WBB was a valid tool for assessing standing balance in elderly subjects. It is entirely plausible that the WBB could be integrated into community practice, although clinical protocols and software would have to be developed to exploit this accurate hardware. Longitudinal studies are currently in progress to investigate the WBB and associated games as a balance rehabilitation tool.

\section{Challenges for Adoption}

\section{Scientific Validation}

Evidence-based medicine (clinical and/or cost-benefit) is the cornerstone of acceptance and uptake of all new forms of clinical intervention. Before any new methodology or technology is applied into the health care domain it must be rigorously validated. To validate a technology, one must first validate the sensor and the algorithms used to convert the sensor data to gait and balance parameters against a gold-standard measure. They must then, as with all new clinical assessments, measure the new parameters on a sufficiently large, randomly controlled group to identify which parameters significantly discriminate between the test group (fallers) and control group (non-fallers). Randomized controlled trials (RCTs) remain the gold standard test of efficacy among clinicians and health-service decision makers. However, RCT's are expensive and a prospective RCT with a fall or fracture as an outcome would require a multi-year trial monitoring hundreds of older adults.

From a research perspective, applying low-cost risk assessments into a wider community setting would generate highly valuable in-situ longitudinal information on older adults; a broad dataset which would be very expensive to replicate in a clinical RCT. It is expected that prospective longitudinal studies in community settings will improve our understanding of falls and the progression of falls risk, and provide the supporting evidence to underpin the accuracy of falls-risk estimation. Relevant findings could be applied to improve the standardized processes and decision-support systems for falls risk management. These data would also be 
unique, in that they are captured in the community for application in the community, thus reducing the "traditional separation between the generation and application of medical knowledge", referred to by Bohmer (2010) in a recent Harvard Business review article. Currently, researchers generate retrospective clinical knowledge about high-risk fallers in a clinical setting, which after an extended period of time and through various avenues appears on a curriculum for clinical practitioners.

Clinical Pathways

Poor service integration and resourcing and the absence of standardized identification and assessment procedures were highlighted as barriers to the provision of effective falls prevention services

(Source: HSE Falls and Fracture Prevention (2010))

Identification of risk is of limited value if there is no clear clinical pathway to act on the identified risk. Currently, community-based practitioners have limited knowledge of the presence of falls clinics. Those who are aware of these specialist services only refer those who have experienced an injurious fall, recurrent falls or have obvious gait or balance impairment. If the various falls prevention algorithms are to be implemented in full, community-based practitioners must be aware of the services provided by falls clinics, where they are located, and what the referral process is to access them. In implementing these pathways, falls clinics will receive more referrals from the community, including referrals from lower risk fallers than were previously referred to them. It is therefore critical that falls clinic staff, who will be impacted by these changes, are "bought into" the validity of community-based sensor based risk assessment and do not see them as unnecessarily adding to their workloads.

New clinical pathways will necessitate the transfer of patient records from the community to a falls clinic, perhaps to a specialist unit such as physiotherapy, and back to the community. Personal Health Records (PHRs) can provide an important tool for the gathering, collation and archiving of personal health information. They can provide an important conduit of information from the primary facility to acute care setting where specialized assessments are provided. Secondly they can provide an improved link between the findings of the specialized assessments and the subsequent care which must be provided back in the community once the patient has returned home. In adopting PHRs for falls clinical pathways, each assessing clinician would have full access to the faller's longitudinal gait and balance history in the community, results from falls clinic assessments, prescribed interventions, and the longitudinal impact of these interventions on the faller's gait and balance parameters as recorded in the community. Access to this data, would reduce unnecessary repetition of lengthy interviews and assessments thus enabling more efficient use of clinical resources.

\section{Cost and Reimbursement}

Implementing a gait and balance community-screening program using clinical assessment tools would be prohibitively expensive. Fortunately, competition in the 
mobile phone and computer-gaming sectors have resulted in the rapid evolution of low-cost, low-power, wireless, sensing, and processing technologies. These technologies have been applied in BSNs to cheaply and accurately measure large movements such as gait, and small movements such as those made to maintain balance. Thus, accurate gait and balance assessment systems are sufficiently inexpensive for community-based practitioners. However, when various forms of technology are introduced, the question of who is going to pay for the technology and service is often raised. End user and clinicians are unwilling to forego out-ofpocket expense. Yet, there is an obvious cost advantage that can be met by providing early falls-risk assessments in the community. Falls-clinic services will also require additional resources if all those with gait and balance impairments are referred from the community to falls clinics. It is therefore critical that the cost-benefit analysis of changing from reactionary to preventive models of care will have to be clearly demonstrated to decision-makers. The issue of cost needs to be addressed into two ways. One requires support at government level by establishing polices that support the provisioning of the technology, training, and assessment costs. Secondly, health care insurers need to be incentivized to cover reimbursement costs. The value proposition around advocating and reimbursing regular risk-assessment evaluations needs to developed for insurers to buy into the process.

\section{IT Barriers}

A community-screening program must be scalable to assess as many people as possible; thus, it is essential that screening measures are both accurate and repeatable. Accuracy ensures that correct conclusions are drawn from the data and longitudinal trends are valid. Repeatable measurements ensure that the same results are obtained regardless of the person administrating the test, the location of the test, or the date of the test. Validated sensor-based measurement and a clear protocol can provide both accuracy and repeatability that are difficult to achieve with observation and/or scales.

Configuring, using, and interpreting sensor data requires a high level of technical skill and training in digital-signal processing, which very few, if any, community doctors have. It is therefore imperative that interaction with the sensors is limited to simply attaching them and initiating and terminating data capture. Other sensor tasks, such as installation, configuration, data transfer, and data analysis, should therefore be automated or as user friendly as possible. Gait and balance parameters, such as sway, stride length, step width; which are calculated from the sensor data, can be meaningless to all but gait and balance experts. Thus, simply providing a list of parameters to a community-based doctor, without explaining what each parameter represents, will render the technology useless to the doctor and will negatively affect uptake and compliance with the technology. It is therefore important that the technology not only explains what each parameter represents, but also informs the doctor how these parameters compare to age-matched "normal" individuals. To be truly valuable to doctors, these results should also be interpreted using current research findings to provide diagnostic indicators of pathologies, such as Parkinson's or falls risk.

Implementing a sensor-based system in a community setting requires not only accurate and reliable technology; it must also integrate seamlessly into the doctor's 
existing IT infrastructure. In the doctor's office, the system must interface with hardware, such as PCs and printers, and software, such as patient record software. Data gathered in the community must be transferred along the clinical pathway for referral; thus, an electronic method of transferring data will be required if an integrated IT infrastructure exists, and a paper-based method will be required if such infrastructure does not exist. If community-acquired data is to be collated into a central repository for the purposes of longitudinal research, secure communication methods, patient anonymity, and data encryption must also be considered.

\section{Designing Technology for the Community}

Things occur in time and place; this means that technologies need to be understood as "systems" in order to be successfully implemented (Prendergast and Roberts 2009). If we want to contribute to the decrease of falls in older adults, we need to think not only to "what" type of technology could help, but also to "when", "where", "how", by "whom" and "why" this technology would be used. Therefore, to create a technologically improved version of a traditional falls-risk assessment test, we need to learn about the system in which it is currently used. We would need to know who would use it, what characteristics it should have, the space it should be implemented in, and so on. Current clinical technologies for falls-risk assessments need specific spaces in order to be performed, and expertise to interpret the results; yet to truly tackle falls we need population-based technologies that are reliable, scalable, and easy to use. These technologies also need to do more: They need to be better than traditional methods and current technologies. They need to be perceived as useful by practitioners and potential patients, and not threatening or challenging of their expertise.

Ethnography provides an insight into complex social and cultural context that surround problems and technologies as experienced in everyday life. Ethno-driven design ensures that products are not designed in isolation from their social context; rather, products are developed within the system in which they will be implemented. A user-centered design approach includes the perspectives of a potential user throughout its development (Brown 2008), thus ensuring that the end-product fits the requirements of the user. Research in the technology and health domains involves at least three different spheres of knowledge: clinical practice, engineering, and product design. These three different disciplines have different languages, priorities, and values. To successfully create a technological version of an existing test, input is required from multiple disciplines throughout the development cycle. Clinical experience is required to identify an appropriate test; engineering experience is required to identify suitable sensors and develop measures; and product design is required to translate engineering measures into a product which the user wants and can use. Ethnography provides deeply textured contexts of research needs and methods by gathering and challenging the point of view of the different disciplines involved in its production.

To automate measurement of a gait and balance test, such as the Timed Up and Go (Fig. 2), the multidisciplinary team must not only establish what the standardized protocol for the assessment is, but also the subliminal evaluations which the assessor makes. These may include: how long the person being assessed took to rise from the 

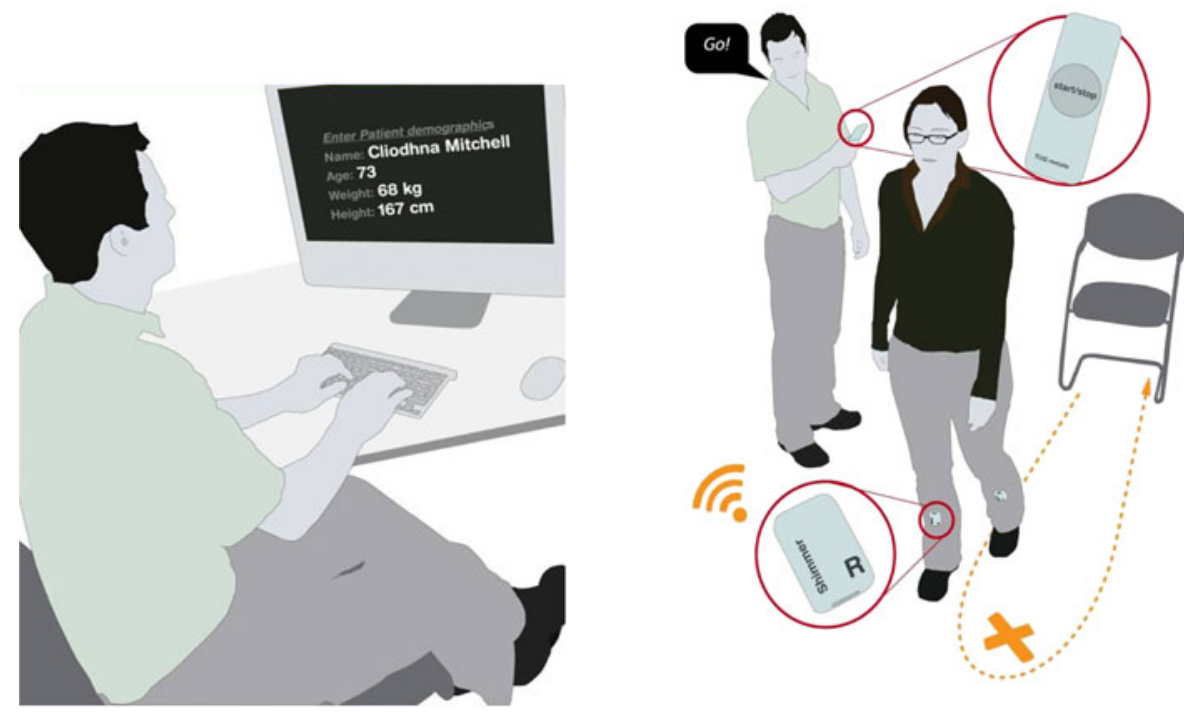

Fig. 2 Illustration of a BSW implementation of the Timed Up and Go test, in which a doctor enters a patient's demographic details and then administers test

chair; if they stumbled during the walk; the fluidity of the turn; and how they sat into the chair. In challenging a clinician's perspective of how they administer and interpret a test, one can gather a richer view of what aspects are most meaningful from a clinical perspective, and transfer this knowledge to the engineers, who can select appropriate hardware and software to capture data and develop algorithms to quantify the required parameters. In analyzing the data, the engineers can add further value by identifying additional parameters, which would be impossible to measure through observation alone. Statistical methods can then be applied to a sufficiently large dataset to identify which, if any, of the derived parameters significantly discriminate between the case and control groups. Product designers then work with the multidisciplinary team and potential end-users to iteratively develop prototypes that best meet the needs of the end-user. Each iteration must be evaluated until a prototype, which fulfils accuracy, cost, usability, and interoperability criteria, is obtained.

\section{Conclusion}

Falls present significant financial and societal problems to both the faller and health care systems. Current reactive approaches, which simply assess falls risk following a fall event, are unsustainable. Early detection of falls risk is necessary to maximize the benefit of intervention. New models of care have been proposed that move the assessment of falls risk from expensive clinical-centric models only available to those who live near a falls clinic and are at high risk of falling, to cheaper community-based approaches, available to all. However, the screening methods proposed by these models involve detailed interviewing and implementation of either time-consuming, subjective ordinal scales or quicker, non-diagnostic functional scales. Technology can provide accurate and repeatable assessments, but the 
majority of existing technology is costly, time-consuming, and may require expert knowledge, expensive equipment, and specialized spaces to perform the tests. Current research and emerging commercial products indicate that BSNs are equivalent or better than existing scales in measuring falls risk. These low-cost, easy-to-use, flexible systems can quickly and objectively measure a range of gait and balance parameters; thus, they appear to be ideal for assessment of falls risk in a community setting.

Community-based medical practices have different work-flows, time constraints, resources, and knowledge levels to specialized clinics, thus different technology is required in the community. Ethnography and user-centered design methods can be applied to better understand the various technical and usability requirements for a falls-risk assessment solution. To be valuable to the community-based doctor, the technology must be accurate, reliable, and repeatable; the results should be interpreted; and referral pathways should be proposed. Longitudinal data gathered in the community using accurate technology can also extend our knowledge of development and progression of falls risk. Technology can therefore not only improve the implementation of existing falls guidelines by enabling early detection and intervention of falls risk, but it may potentially provide evidence to develop new guidelines and enable better prediction and prevention of falls.

Acknowledgements This research was completed as part of a wider program of research within the TRIL Centre, (Technology Research for Independent Living). The TRIL Centre is a multi-disciplinary research centre, bringing together researchers from UCD, TCD, NUIG \& Intel, funded by Intel, IDA Ireland and GE Healthcare. www.trilcentre.org

Open Access This article is distributed under the terms of the Creative Commons Attribution Noncommercial License which permits any noncommercial use, distribution, and reproduction in any medium, provided the original author(s) and source are credited.

\section{References}

American Geriatric Society, British Geriatics Society, and American Academy of Orthopaedic Surgeons Panel on Falls Prevention (2009) Guideline for the Prevention of Falls in Older Persons. Available via American Geriatric Society. http://www.medcats.com/FALLS/frameset.htm. Cited 20 September 2010.

Aminian, K., Trevisan, C., Najafi, B., Dejnabadi, H., Frigo, C., Pavan, E., et al. (2004). Evaluation of an ambulatory system for gait analysis in hip osteoarthritis and after total hip replacement. Gait \& Posture, 20(1), 102-107.

Aminian, K., Najafi, B., Büla, C., Leyvraz, P. F., \& Robert, P. (2002). Spatio-temporal parameters of gait measured by an ambulatory system using miniature gyroscopes. Journal of Biomechanics, 35(5), 689-699.

AMTI Force Platforms (2010). Available via Advanced Mechanical Technology Inc. http://amti.biz/ Platformhome/PlatformHome.htm. Cited 20 September 2010.

Berg, K., Wood-Dauphinee, S., Williams, J. I., \& Gayton, D. (1989). Measuring balance in the elderly: preliminary development of an instrument. Physiotherapy Canada, 41, 304-331.

Biodex Fall Screening and Conditioning Program (2010). Available via Biodex Medical Systems Inc. http://www.biodex.com/rehab/fallprogram/fallprogram.htm. Cited 20 September 2010.

Bohmer, R. M. J. (2010). Fixing health care on the front lines. Harvard Business Review, 88(4), 62-69.

Brown, T. (2008). Design thinking. Harvard Business Review, 85-92.

Codamotion (2010). Available via Charnwood Dynamics Ltd. http://www.codamotion.com/. Cited 20 September 2010.

Clark, R. A., Bryant, A. L., Pua, Y., McCrory, P., Bennell, K., \& Hunt, M. (2010). Validity and reliability of the Nintendo Wii Balance Board for assessment of standing balance. Gait \& Posture, 31(3), 307-310. 
Davis, J. C., Robertson, M. C., Ashe, M. C., Liu-Ambrose, T., Khan, K. M., \& CA, Marra. (2010a). Does a home-based strength and balance programme in people aged $>$ or $=80$ years provide the best value for money to prevent falls? A systematic review of economic evaluations of falls prevention interventions. British Journal of Sports Medicine, 44(2), 80-89.

Davis, J. C., Robertson, M. C., Ashe, M. C., Liu-Ambrose, T., \& Khan, K. M. (2010b). International comparison of cost of falls in older adults living in the community: A systematic review. Osteoporosis International, 21(8), 1295-1306.

Duncan, P. W., Weiner, D. K., Chandler, J., \& Studenski, S. (1990). Functional reach: a new clinical measure of balance. Journal of Gerontology, 45, M192-M197.

Dynaport Gait Test (2010). Available via McRoberts B.V. http://www.mcroberts.nl/products/clinician/ dynaport-gaittest. Cited 20 September 2010.

DynaPort SwayTest (2010). Available via McRoberts B.V. http://www.mcroberts.nl/products/clinician/ dynaport-swaytest. Cited 20 September 2010.

Fitzpatrick, C., Simpson, J. M., Valentine, J. D., Ryder, S., Peacock-Edwards, T., Sidnell, P., et al. (2005). The measurement properties and performance characteristics among older people of TURN180, a test of dynamic postural stability. Clinical Rehabilitation, 19(4), 412-418.

GAITRite M2 (2010). Available via CIR Systems Inc. http://www.gaitrite.com/. Cited 20 September 2010.

GAITRite Walkway (2010). Available via CIR Systems Inc. http://www.gaitrite.com. Cited 20 September 2010.

Giansanti, D. (2006). Investigation of fall-risk using a wearable device with accelerometers and rate gyroscopes. Physiological Measurement, 27, 1081-1090.

Gillespie, L. D., Robertson, M. C., Gillespie, W. J., Lamb, S. E., Gates, S., Cumming, R. G., Rowe, B. H. (2009). Interventions for preventing falls in older people living in the community. Cochrane Database of Systematic Reviews 2009, Issue 2. Art. No.: CD007146. doi:10.1002/14651858.CD007146.pub2.

Greene, B. R., O’Donovan, A., Romero-Ortuno, R., Cogan, L., Scanaill, C. N., \& Kenny, R. A. (2010). Falls risk assessment through quantitative analysis of TUG. Paper presented at the 1st AMA-IEEE Medical Technology conference on Individualised Healthcare.

Guidelines commissioned by the National Institute for Clinical Excellence (NICE). (2004). Falls: Assessment and Prevention of Falls in Older People. Published by the Royal College of Nursing, London.

Horak, F. B., Wrisley, D. M., \& Frank, J. (2009). The Balance Evaluation Systems Test (BESTest) to differentiate balance deficits. Physical Therapy, 89(5), 484-498.

Kannus, P., Palvanen, M., Niemi, S., \& Parkkari, J. (2007). Alarming rise in the number and incidence of fall-induced cervical spine injuries among older adults. Journals of Gerontology. Series A, Biological Sciences and Medical Sciences, 62(2), 180-183.

Kenny, R. A., Romero-Ortuno, R., \& Cogan, L. (2009). Falls. Medicine, 37(2), 84-87.

Masud, T., \& Morris, R. O. (2001). Epidemiology of falls. Age Ageing, 30(suppl_4), 3-7.

Najafi, B., Aminian, K., Loew, F., Blanc, Y., \& Robert, P. (2002). Measurement of stand-sit and sit-stand transitions using a miniature gyroscope and its application in fall risk evaluation in the elderly. IEEE Transactions on Biomedical Engineering, 49(8), 843-851.

Narayanan, M. R., Redmond, S. J., Scalzi, M. E., Lord, S. R., Celler, B. G., \& Lovell Ast, N. H. (2010). Longitudinal falls-risk estimation using triaxial accelerometry. IEEE Transactions on Biomedical Engineering, 57(3), 534-541.

NeuroCom EquiTest ${ }^{\circledR}$ (2010). Available via NeuroCom International Inc. http://www.resourcesonbalance. com/neurocom/products/EquiTest.aspx. Cited 20 September 2010.

Novel Pedar ${ }^{\circledR}$ (2010). Available via Novel GmbH. http://novel.de/novelcontent/pedar. Cited 20 September 2010.

O’Sullivan, M., Blake, C., Cunningham, C., Boyle, G., \& Finucane, C. (2009). Correlation of accelerometry with clinical balance tests in older fallers and non-fallers. Age and Ageing, 38, 308-313.

Podsiadlo, D., \& Richardson, S. (1991). The timed "Up \& Go": a test of basic functional mobility for frail elderly persons. Journal of the American Geriatrics Society, 39(2), 142-148.

Prendergast, D., \& Roberts, S. (2009). Practice, systems and technology for seniors. Universal Access in the Information Society archive, 8(1), 59-61.

Salarian, A., Russmann, H., Vingerhoets, F. J. G., Dehollain, C., Blanc, Y., Burkhard, P. R., et al. (2004). Gait Assessment in Parkinson's Disease: Toward an Ambulatory System for Long-Term Monitoring. IEEE Transactions on Biomedical Engineering, 51(8), 1434-1443.

Shumway-Cook, A., Brauer, S., \& Woollacott, M. (2000). Predicting the probability for falls in community-dwelling older adults using the timed up \& go test. Physical Therapy, 80(9), 896-903.

Skrba, Z., O’Mullane, B., Greene, B. R., Ni Scanaill, C., Fan, C. W., Quigley, A., et al. (2009). Objective real-time assessment of walking and turning in elderly adults. Paper presented at the Annual International Conference of the IEEE Engineering in Medicine and Biology Society. 
Strategy to Prevent Falls and Fractures in Ireland's Ageing Population. Available via Health Service Executive. http://www.hse.ie/eng/services/Publications/services/Older/Strategy_to_Prevent_Falls_ and Fractures in Ireland\%E2\%80\%99s Ageing Population.html. Cited 20 September 2010.

Tekscan F-Scan ${ }^{\mathbb{B}}(2010)$. Available via Tekscan Inc. http://www.tekscan.com/medical/system-fscan1.html. Cited 20 September 2010.

Tekscan MatScan ${ }^{\mathbb{B}}$ (2010). Available via Tekscan Inc. http://www.tekscan.com/medical/system-matscan1. html. Cited 20 September 2010.

Tekscan Sway Analysis Module ${ }^{\mathrm{TM}}$ (2010). Available via Tekscan Inc. http://www.tekscan.com/medical/ sway-analysis.html. Cited 20 September 2010.

Tinetti, M. E. (1986). Performance-orientated assessment of mobility problems in elderly patients. JAGS, 34, 119-126.

Tinetti, M. E., Richman, D., \& Powell, L. (1990). Falls efficacy as a measure of fear of falling. Journal of Gerontology, 45(6), 239-P243.

Tyson, S. F., \& Connell, L. A. (2009). How to measure balance in clinical practice. A systematic review of the psychometrics and clinical utility of measures of balance activity for neurological conditions. Clinical Rehabilitation, 23(9), 824-840.

United Nations Department of Economic and Social Affairs, Population Division (2009). World Population Ageing 2009.

Zijlstra, W., \& Aminian, K. (2007). Mobility assessment in older people: new possibilities and challenges. European Journal of Ageing, 4(1), 3-12.

Cliodhna Ní Scanaill Ph.D is a Falls Research Engineer in Intel Labs, Health Research and Innovation team in Leixlip, Ireland. She worked on the Falls Prevention strand of the TRIL Centre for over 3 years, as a software engineer, researcher, and Principal Investigator. Her research interests include falls, gait analysis, wireless sensor networks, telemonitoring, and smart home technology. Prior to joining Intel in 2006, she completed a PhD, titled "Remote Mobility Monitoring of the Elderly using SMS messaging", in the Biomedical Electronics Laboratory, University of Limerick, Ireland. Cliodhna holds a B.Eng in Computer Engineering and a Ph.D. in Biomedical Electronics from the University of Limerick.

Chiara Garattini Ph.D is a Post Doctoral Research Fellow in Ethnography and Design at the TRIL Centre. She received her Ph.D. in Anthropology at the National University of Ireland - Maynooth. Dr Garattini's research focuses on ageing, technology, independent living, death and chronic illnesses. Currently she is contributing at different extents to all the strands and projects within the TRIL Centre where qualitative research is employed, interacting with an array of researchers from such diverse disciplines as engineering and neuroscience. In particular, she contributes to different stages of the technology design process and has a particular research interest in gaining deeper insights in the way users (being health professionals, patients or other) understand and interact with technology. She also contributes to the deployment of these technologies in home trials, where she explores the challenges faced when technology is translated from the lab to the real world.

Barry R. Greene Ph.D is a biomedical engineer with Intel labs and the TRIL Centre working independent living technologies. Prior to joining Intel in May 2008, he worked as a postdoctoral fellow in University College Cork developing automated neonatal seizure detection algorithms. He received the $\mathrm{BE}$ (Electronic) and Ph.D. degrees from University College Dublin in 2003 and 2007 respectively. His doctoral thesis was entitled 'Quantification and Classification of Electrophysiological markers of seizure in the neonate'. His research interests are in clinical applications of biomedical signal processing.

Michael McGrath Ph.D is a senior technologist in the Health Research and Innovation group. Michael is also a principal investigator at the Technology Research for Independent Living (TRIL) Centre focusing on the development technologies to support independent living research. His areas of interest include sensors, wireless communications, assisted living technologies, intelligent user interfaces, data fusion and data management techniques. Michael co-authored the 2009 book Wireless Sensor Networks for Healthcare Applications. Michael received his B.Sc. in Analytical Science and Ph.D. in sensors and instrumentation from Dublin City University. Michael also holds a graduate diploma in Information Technology from Dublin City University, a Graduate Diploma and Master's Degree in Computing from ITB. Michael is a Charted Chemist (CChem) and a Charted Scientist (CSi). 